\title{
Festrede „10 Jahre zfhr“
}

Online publiziert: 21. Februar 2012

(C) Springer-Verlag 2012

\section{Sehr geehrte Festgäste!}

Herzlich willkommen auch im Namen der Herausgeber, für die ich stellvertretend einige Worte an Sie richten darf.

Ich möchte zunächst meiner Freude darüber Ausdruck verleihen, dass unsere heutige Feier so breit gefächerten Zulauf, aus einer ganzen Reihe hochschulischer und universitärer Einrichtungen, gefunden hat.

So haben wir nicht nur Vertreter verschiedener wissenschaftlicher und künstlerischer Universitäten in Wien, Graz, Salzburg, Linz und Krems unter uns, sondern auch Angehörige verschiedenster zentraler hochschulischer Institutionen, wie des Bundesministeriums für Wissenschaft und Forschung, der Österreichischen Universitätenkonferenz, der Österreichischen Fachhochschulkonferenz, des Österreichischen Fachhochschulrates, des Zentralausschusses der Universitätslehrerinnen und Universitätslehrer sowie der Österreichischen Hochschülerinnenund Hochschülerschaft. Und schließlich können wir auch die Hochschulpolitik, in Person des Bildungssprechers der Österreichischen Grünen, bei uns begrüßen.

Dieses vielfältige Interesse maßgeblicher „Player" der österreichischen Hochschullandschaft am Festtag unserer Zeitschrift, darf ich für uns Herausgeber als ermunterndes Zeichen des Zuspruchs werten.

Es ist uns auch eine Ehre, dass es gelungen ist, mit den Professoren Löwer und Mittelstra $\beta$ zwei hochkarätige Vortragende für das Symposium zu gewinnen.
Nun, Jubiläen, wie das heute gefeierte, sind immer auch ein Anlass für Rückblicke auf die Entstehung des Gewordenen.

Und wenn man an die Geburtsstunde der Zeitschrift für Hochschulrecht denkt, so ist sie zunächst maßgeblich mit der Person Werner Hauser verbunden, der ja nicht nur der eigentliche Initiator des Projekts „zfhr" war, sondern auch die für die Ermöglichung seiner Verwirklichung erforderlichen Finanzquellen lukrierte. Bis zu seinem freiwilligen Ausscheiden, hat er die Zeitschrift stets mit vollem Einsatz und viel Akribie begleitet.

An dieser Stelle ist auch Mario Kostal hervorzuheben, der gewissermaßen der Zweite im Bunde des von Gerald Muther einst als „Triumvirat" bezeichneten Gründerteams war. Mit seinem Witz und seinem Charme hat er den nicht wenigen Gründungsverhandlungen und -sitzungen immer wieder Lebensfrohheit eingehaucht.

Das „Triumvirat" hat ja zwischenzeitig fast schon zu einem „Feminat“ mutiert. Und so darf ich mich heute an der Seite von Bettina PertholdStoitzner und Eva Schulev-Steindl meiner Herausgebertätigkeit erfreuen. Jedenfalls in diesem Bereich ist es damit gelungen, die Frauenquote mehr als zu erfüllen.

Der Erfolg hat ja bekanntlich viele Väter und natürlich auch Mütter. Vorrangig sind dies die Fiancies der Zeitschrift, namentlich die FH-Joanneum in Graz sowie die Fachhochschulkonferenz und die Universitätenkonferenz in Wien. Sie haben bis heute selbstlos die erforderlichen Sponsorgelder für die zfhr zur Verfügung gestellt und damit deren eigentliche Existenzgrundlage geschaffen. In Zeiten vermehrter Sparkurse ist 
dies durchaus keine Selbstverständlichkeit und kann daher nicht genug bedankt werden. Sie sind, wenn man einen anatomischen Vergleich ziehen wollte, das eigentliche Blut in den Adern der Zeitschrift, ohne deren Geldflüsse die zfhr nicht lebensfähig wäre. Ihnen gilt daher an dieser Stelle ein besonderes Dankeschön, verbunden mit der Hoffnung, dass sie uns auch künftighin zur Seite stehen mögen.

Nach verschiedenen Anläufen bei mehreren Verlagen sowie unterschiedlichen Meinungshaltungen und Konzeptionen, ist die Zeitschrift schließlich beim Springer-Verlag gelandet, der bis heute, insbesondere vermittels der Person des Alois Sillaber, eine gute Zusammenarbeit ermöglicht hat. Dass diese Veranstaltung nicht nur von Springer geleitet, sondern auch finanziell zum guten Teil unterstützt wird, ist ein Zeichen dieser Stimmigkeit. Wobei man sich seitens der Herausgeber nur wünschen kann, dass die bewährte Wiener Verlagslinie nicht zu sehr durch Globalisierungseffekte verwässert wird.

Eine erfolgreiche Zeitschrift benötigt immer auch einen renommierten und einsatzfreudigen Beirat. Einen solchen zu finden ist bei der zfhr gut geglückt. Der ursprünglich aus den drei Mitgliedern: Berka, Brünner und Funk bestandene Beirat ist bis heute zu einem Gremium von einem guten Dutzend an Mitgliedern angewachsen, welche bei den jährlichen Beirattreffen nicht nur zahlreich erscheinen, sondern immer wieder auch wesentliche Inputs für die künftige Ausrichtung der Zeitschrift geliefert haben. Schließlich ist ja selbst die Idee zu dieser Jubiläumsfeier anlässlich einer Beiratssitzung geboren worden.

Die Quintessenz jeder Zeitschrift sind natürlich die Autoren, die, wenn man die Anatomie noch einmal bemühen will, das Gehirn der zfhr darstellen, ohne deren Gedanken es Medien, wie dieses, nicht bräuchte. Über die Jahre hat sich für die zfhr dabei ein erfreulicher Zulauf von Autorinnen und Autoren ergeben, der auch das Interesse der Autorenlandschaft an hochschulrechtlichen Themen widerspiegelt.

Last but not least ist Generalsekretär Faulhammer, als Hausherrnvertreter, der ja auch Angehöriger des Beirates ist, für die spontane und unkomplizierte Bereitschaft zur Zurverfügungstellung von Räumlichkeiten und finanzieller Unterstützung besonders zu danken.

All den Genannten sei, im Namen der Herausgeber, nochmals ein herzliches Danke für ihr Engagement und ihre Unterstützung ausgedrückt.

Und ich darf abschließend der Zeitschrift und allen, die an ihrem Werden beteiligt sind, noch viele interessante und fruchtbare Jahre wünschen. - Ad multos annos!

Ass.-Prof. Mag. Dr. Manfred Novak 